\title{
Postnatal Maturation of the Red Nucleus Motor Map Depends on Rubrospinal Connections with Forelimb Motor Pools
}

\author{
Preston T. J. A. Williams, Sangsoo Kim, and John H. Martin \\ Department of Physiology, Pharmacology and Neuroscience, City College of the City University of New York, New York, New York 10031
}

\begin{abstract}
The red nucleus (RN) and rubrospinal tract (RST) are important for forelimb motor control. Although the RST is present postnatally in cats, nothing is known about when rubrospinal projections could support motor functions or the relation between the development of the motor functions of the rubrospinal system and the corticospinal system, the other major system for limb control. Our hypothesis is that the RN motor map is present earlier in development than the motor cortex (M1) map, to support early forelimb control. We investigated RN motor map maturation with microstimulation and RST cervical enlargement projections using anterograde tracers between postnatal week 3 (PW3) and PW16. Microstimulation and tracer injection sites were verified histologically to be located within the RN. Microstimulation at PW4 evoked contralateral wrist, elbow, and shoulder movements. The number of sites producing limb movement increased and response thresholds decreased progressively through PW16. From the outset, all forelimb joints were represented. At PW3, RST projections were present within the cervical intermediate zone, with a mature density of putative synapses. In contrast, beginning at PW5 there was delayed and age-dependent development of forelimb motor pool projections and putative rubromotoneuronal synapses. The RN has a more complete forelimb map early in development than previous studies showed for M1, supporting our hypothesis of preferential rubrospinal rather than corticospinal control for early movements. Remarkably, development of the motor pool, not intermediate zone, RST projections paralleled RN motor map development. The RST may be critical for establishing the rudiments of motor skills that subsequently become refined with further CST development.
\end{abstract}

Key words: corticospinal tract; development; motor neuron; red nucleus; rubrospinal tract; spinal cord

\section{Introduction}

Skilled limb movements are the last motor abilities to be mastered during postnatal development. In maturity, motor skills are dependent on the corticospinal tract (CST), originating principally from the motor cortex (M1), and the rubrospinal tract (RST), originating from the red nucleus $(\mathrm{RN})$, which are collectively termed the lateral motor system (Kuypers, 1981). It is widely regarded that the motor commands for distal limb control arise primarily from the descending projections of the lateral system. How the lateral motor system develops to support motor skills by controlling spinal motor circuits remains unclear. Much progress has been made in understanding the importance of the corticospinal system for the development of distal limb control (Porter and Lemon, 1993; Martin et al., 2009). The corticospinal system is late-developing, undergoes important postnatal refine-

Received Dec. 20, 2013; revised Jan. 27, 2014; accepted Feb. 13, 2014

Author contributions: P.T.J.A.W. and J.H.M. designed research; P.T.J.A.W. and J.H.M. performed research; P.T.J.A.W. and S.K. analyzed data; P.T.J.A.W. and J.H.M. wrote the paper.

This work was supported by National Institutes of Health Grants R01NS036835 and R01NS079569 (J.H.M.). We thank Xiuli Wu for help with histochemistry and histology, Dustin Zuelke for help with data analysis, and Dr. Samit Chakrabarty for assistance with the antidromic experiments. We also thank Harry Acosta and Dr. Sulli Popilskis for animal and veterinary care, respectively.

The authors declare no competing financial interests.

Correspondence should be addressed to Dr. John H. Martin, Department of Physiology, Pharmacology and Neuroscience, City College of the City University of New York, 160 Convent Avenue, New York, NY 10031. E-mail: jmartin@ccny.cuny.edu.

DOI:10.1523/JNEUROSCI.5332-13.2014

Copyright $\odot 2014$ the authors $\quad 0270-6474 / 14 / 344432-10 \$ 15.00 / 0$ ment in both humans and animals, and has a rapid development when dexterous limb skills are first being learned and practiced. The importance of the rubrospinal system, the other major lateral motor system for limb control, is less well understood. If corticospinal and rubrospinal systems serve similar functions in maturity, they would be predicted to codevelop to coordinate their motor commands. In the event of an injury producing limb impairments, for example, M1 stroke, the RST could spontaneously compensate and restore limb control (Lawrence and Kuypers, 1968a). Alternatively, if the two components are functionally distinct, then there may be differences in the timing of rubrospinal and corticospinal system development. We hypothesize that the phylogenetically older rubrospinal system (Massion, 1967) develops earlier than the corticospinal system and initially provides the rudiments for distal limb movements. Such a sequential pattern of development implies that the two systems contribute unique motor commands, and would help explain why spontaneous recovery from injury is most often incomplete.

We examined the RN motor representations and RST projections to the cervical enlargement from early in postnatal development to adulthood in cats. We were particularly interested in studying the relationship between the RN motor map and the RST projections to forelimb motor pools, indicating direct connections with motoneurons (Holstege, 1987; McCurdy et al., 1987). Early development of rubromotoneuronal terminations, before distal limb skills are expressed, implies a role in muscle control, but not necessarily in motor skill. It has been demon- 
strated in monkeys that the timing of corticomotoneuronal development overlaps with the period when relatively independent finger movements are expressed ( $\sim 6$ months; Porter and Lemon, 1993). Because cats do not have corticomotoneuronal terminations, it would be intriguing if the rubromotoneuronal connections were established as skilled limb movements develop.

We show, for the first time, that the $\mathrm{RN}$ has a more complete forelimb motor map, especially for the distal joints, earlier in development than previous studies showed for M1. Remarkably, development of RST projections to the motor pools, not to the intermediate zone, paralleled RN motor map development, and this occurred during the postnatal period that coincides with the transition to adaptive motor skills. These findings support our hypothesis that the rubrospinal system is more important than the corticospinal system for control of early movements.

\section{Materials and Methods}

Subjects and timeline. All experimental procedures were approved by and conducted in accordance with the Institutional Animal Care and Use Committee of the City College of the City University of New York. Cats were obtained from a supplier accredited by the Association for Assessment and Accreditation of Laboratory Animal Care International and housed in a controlled vivarium with food and water available ad libitum. Cats were delivered in litters of four, with a lactating adult female, 1 week before the age required for experiments. We used the minimum number of animals necessary to complete the studies, and care was taken to reduce any pain or discomfort throughout all experiments.

General surgical procedures. For all surgical procedures, cats were given a broad-spectrum antibiotic (cefalozin; $25 \mathrm{mg} / \mathrm{kg}$, i.m.) and, to reduce oral secretions, atropine $(0.04 \mathrm{mg} / \mathrm{kg}$, i.m. $)$. Anesthesia was induced with a mixture of acepromazine $(0.03 \mathrm{mg} / \mathrm{kg}, \mathrm{i} . \mathrm{m}$. $)$ and ketamine hydrochloride $(30 \mathrm{mg} / \mathrm{kg}$, i.m.). Fluids were supplemented with lactated Ringer's solution. All survival surgeries were performed under aseptic conditions, and vital signs were monitored to ensure an appropriate level of anesthesia. For survival surgeries (tracer injections), animals were intubated and anesthesia maintained with $1.5-3 \%$ isoflurane in oxygen. Animals were kept in a warming incubator until they recovered from anesthesia and then were returned to their home cage. Kittens typically resumed nursing within 4-6 h after recovering from anesthesia and were orally supplemented with feline replacement milk (KMR brand) as needed. Immediately following surgery, cats were administered antibiotics (cefazolin; 25 $\mathrm{mg} / \mathrm{kg}$, i.m.) and analgesics (buprenorphine, $0.02 \mathrm{mg} / \mathrm{kg}$, i.m.; or carprofen, $2 \mathrm{mg} / \mathrm{kg}$, i.m.) twice daily for $2 \mathrm{~d}$ after surgery, or longer if necessary. For terminal experiments (intracortical microstimulation), anesthesia was maintained with continuous infusion of ketamine $(10 \mathrm{mg} / \mathrm{kg} / \mathrm{h}$, i.v. $)$ as in our previous studies (Friel and Martin, 2007).

Microstimulation mapping. We used microstimulation to determine the efficacy of rubrospinal projections to evoke forelimb movements throughout the postnatal period. Kittens and adults were induced for anesthesia as above and maintained areflexive with continuous intravenous ketamine infusion. For each animal, the head was fixed in a stereotaxic frame, and craniotomies over the right and left sides of the midbrain were drilled (anteroposterior, 0-5; mediolateral, 0-3.5; Berman, 1968). Electrode tracks and marking lesions were reconstructed histologically to verify that microstimulation sites were within the red nucleus.

In an effort to capture a detailed map of the red nucleus, we recorded evoked movements using high-resolution sampling at $200 \mu \mathrm{m}$ depth intervals across multiple anteroposterior and mediolateral origins. The electrode was attached to a micromanipulator and slowly lowered perpendicular to the cortical surface to target the RN. Microstimulation was applied through Paralene-insulated stainless-steel microelectrodes (Microprobe; $0.5 \mathrm{M} \Omega$ nominal impedance, $1-2 \mu \mathrm{m}$ tip). For all ages, stimuli (45-ms-duration train, $330 \mathrm{~Hz}, 0.2 \mathrm{~ms}$ biphasic balanced pulses, with a leading cathodal pulse) were delivered once every $2 \mathrm{~s}$ using a constant current stimulator (A-M Systems). These parameters are routinely used in adults (Asanuma and Sakata, 1967) and have been reported to be effective in young cats (Bruce and Tatton, 1980; Chakrabarty and Martin,
2000). In determining the threshold and topography of microstimulation effects, we kept the limb in a prone posture. Often the limb was stabilized at a proximal joint during stimulation to verify that distal joint movement was not due to an inertial interjoint interaction. When this was done, or whenever the limb was moved, we waited several seconds before microstimulating to minimize the effect of mechanical limb stimulation on current threshold. We characterized movements at the shoulder (retraction, protraction, abduction, adduction), elbow (flexion, extension), wrist (dorsiflexion, plantar flexion, supination, pronation), and at digit joints (dorsiflexion/digit closure, plantar flexion).

We determined the current threshold at effective sites, defined as the lowest current that consistently produced a motor effect. At first, we rapidly raised the current from zero to suprathreshold values, then reduced the current to below threshold, noting the lowest current at which the effect was present. Next we increased the current and noted when the effect reappeared. If necessary, we repeated this procedure until we were confident of the threshold value. We used a maximal current of $200 \mu \mathrm{A}$ to minimize the likelihood of missing effective sites. It has been estimated that $100 \mu \mathrm{A}$ produces a current spread of $\sim 350 \mu \mathrm{m}$ in cortex (Asanuma and Sakata, 1967), which was an acceptable distance considering the sizes of joint representation zones in cat RN (see Results). Microstimulation above $100 \mu \mathrm{A}$ was used only in the younger animals, when the thresholds were high and the number of effective sites was low. Such currents did not produce lesions. Small marking lesions were made for histological verification by passing negative direct current (single pulse, $1-1.5 \mathrm{~mA}$ for $10 \mathrm{~s}$ ). XYZ coordinates of effective sites were plotted using AutoCAD 2013 for the Macintosh computer (Autodesk). The $z$-axis was referenced the same across ages by use of a zeroing stand set to the anatomical center $(-2.5 \mathrm{~mm}$ ventral to interaural plane) of the mature RN (Berman, 1968).

Rubrospinal axon labeling. For a detailed account of the spinal termination zones of the developing RST, we injected anterograde tracers into the red nucleus. Tracers were injected 2-4 weeks before a terminal electrophysiological procedure depending on the age of the animal. The animal was premedicated with a ketamine/acepromazine mix and intubated for anesthesia maintenance under isoflurane. The head was fixed in a stereotaxic frame and craniotomies made (anteroposterior, 0-4 mm; mediolateral, $0-3.5 \mathrm{~mm}$; depending on age). The final injection coordinates were guided by the ventral location of oculomotor nerve stimulation effects and, in animals older than PW3, by verifying effective limb sites using microstimulation (dorsoventral, 1.5-2.5 mm; depending on age). We used either 10\% biotinylated dextran amine [BDA; 10,000 molecular weight (MW); Life Technologies] or 10\% Alexa Fluor 488 dextran amine (AF488DA; 10,000 MW; Life Technologies). The tracer was slowly infused (Picospritzer; World Precision Instruments) using a micropipette over a period of 3-5 $\mathrm{min}$. The pipette was left in place for an additional 2-3 min at each injection site to minimize diffusion. For each $\mathrm{RN}$, we injected tracer along two tracks separated by about $1 \mathrm{~mm}$ (anteroposterior) and $0.5 \mathrm{~mm}$ (mediolateral) apart. For each track we injected at 2-3 sites with a dorsoventral spacing of 200-300 $\mu \mathrm{m}$ apart; at each site we injected 150-300 $\mathrm{nl}$ of tracer solution. The total amount of tracer for each RN was 900-1200 nl. Injection sites were confirmed to be located within the RN after appropriate transport times with both diaminobenzidine (DAB) histochemistry and immunohistochemistry. We aimed to label as much of the magnocellular RN as possible without having tracer spread beyond its boundaries into the adjoining reticular formation. As a consequence, the caudal pole of RN may not have been as well labeled as the more rostral portion of the magnocellular division. Plots of the maximal density of label indicate there was accurate targeting of the RN with minimal spread to surrounding structures. We also used the presence of labeled axons in the dorsolateral funiculus to confirm efficient transport to the cervical spinal cord.

Motoneuron labeling. There is evidence in cats and other species that the RST projects to the motor pools (Holstege, 1987; McCurdy et al., 1987; Mewes and Cheney, 1991; Küchler et al., 2002). To relate the topography of RST terminations to motor pools, we retrogradely labeled motoneurons in two adult cats by injecting cholera-toxin B (CTB; List Biological Laboratories; unconjugated, low-salt; reconstituted with sterile purified water to a concentration $1 \%$ in $0.01 \mathrm{~m}$ sodium phosphate buffer) in two key muscles surgically exposed: extensor carpi radialis 
(ECR) and biceps brachii. A volume of $25 \mu$ l was slowly injected into multiple intramuscular locations using a Hamilton syringe. The needle was held within the muscle for several minutes after each injection to avoid leakage. We used a survival time of $7 \mathrm{~d}$ to allow retrograde transport of CTB from the muscle to the motoneuron soma.

Histology and tracer histochemistry. Cats were deeply anesthetized (sodium pentobarbital; $30 \mathrm{mg} / \mathrm{kg}$, i.v.) and perfused transcardially with saline [at room temperature (RT)], followed by a solution of $4 \%$ paraformaldehyde at pH 7.4. Heparin was injected (200-500 U, i.v.) at the onset of perfusion. For perfusion, a peristaltic pump was used at a predetermined flow rate that depended on the animal's weight. The total perfusion time was $20-30$ $\min$. The brain and spinal cord were removed, postfixed in the same fixative for $2-3 \mathrm{~h}$, and then transferred to $20 \%$ sucrose in $0.1 \mathrm{M}$ phosphate buffer overnight at $4^{\circ} \mathrm{C}$. Frozen transverse sections $(40 \mu \mathrm{m})$ through the cervical spinal cord (C6-T1) were cut and processed for tracers and markers of interest. Histochemistry for BDA and immunohistochemistry for AF488DA were performed to determine the distribution of labeled RST terminals, and immunohistochemistry was performed for CTB to determine the distribution of motor pools. Coronal sections through the midbrain were cut, and alternate sections were processed for BDA and AF488DA, to determine the location of tracer injection sites, and Nissl stained for assessing RN cytoarchitecture and electrode marking lesions.

For visualization of BDA, sections were incubated in PBS containing the avidin-biotin complex reagent as prescribed by the manufacturer (ABC kit; Vector Laboratories) and 0.2\% Triton X-100 for $2 \mathrm{~h}$ at RT. After rinsing, sections were incubated with the chromogen $\mathrm{DAB}$ (Sigma) for 6-30 min. For AF488DA and CTB, we used a primary antibody to the tracer (rabbit anti-Alexa Fluor 488, 1:400, RT, 6-30 min, Life Technologies; goat-anti CTB, 1:2000, RT, overnight, List Biological Laboratories) and either DAB (secondary; donkey anti-rabbit, 1:200, Vector Laboratories) or fluorescence methods (see next paragraph) for visualization. Each antibody was tested for efficacy for both the DAB and fluorescent methods using a range of primary antibody concentrations. Although is was not possible to coincubate all tissues from cats of different ages, we routinely coincubated tissue from selected pairs of animals to verify that age-related differences were not due to subtle differences in immunohistochemical reactions. For peroxidase staining, sections were dehydrated before coverslipping with mounting medium.

Additional sections were processed for double or triple labeling to visualize the tracers in relation to markers for presynaptic sites as well as for motoneurons positive for choline acetyltransferase (ChAT). The following antibodies were used, at the concentrations and incubation times indicated: (1) monoclonal mouse-anti synaptophysin (SYN; Millipore; SY38, $38 \mathrm{kDa} ; 1: 1000,4^{\circ} \mathrm{C}$, overnight) and (2) polyclonal goat anti-ChAT (Millipore; AB144P; 70-74 kDa; 1:100; $4^{\circ} \mathrm{C}$, overnight). For each primary antibody, free-floating sections were incubated in PBS containing the primary antibody in blocking buffer (3\% donkey serum in $1 \mathrm{M}$ PBS with $0.2 \%$ Tween $20, \mathrm{pH} 7.4$ ). After rinsing, sections were incubated in blocking buffer containing a secondary antibody conjugated to Cy3 (1: 800; RT; $1 \mathrm{~h}$; donkey anti-rabbit, anti-goat, or anti-mouse; Jackson ImmunoResearch Laboratories), to FITC (1:500; RT; 2 h; donkey anti-rabbit, anti-goat, or anti-mouse; Life Technologies), or to Cy5 (1:100; RT; 2 h; donkey anti-goat; abCAM) at $\mathrm{pH}$ 7.4. For fluorescence labeling, sections were mounted on gelatincoated slides, air dried, and coverslipped with Vectashield (Vector Laboratories). We conducted double and triple label immunofluorescence for the following combinations of anterograde tracers and primary antibodies: BDA-AF488DA;
Table 1. Summary of Experiments

\begin{tabular}{lcc}
\hline Age group $^{a}$ & RN stimulation & RST tracing \\
\hline $2-3(6)$ & 4 & 2 \\
$4-6(8)$ & 4 & 5 \\
$7-8(7)$ & 3 & 4 \\
$9-16(7)$ & 6 & 4 \\
Adult (7) & 3 & 4 \\
Total (35) & 20 & 19
\end{tabular}

${ }^{a}$ Age is given in postnatal weeks (group $M$ ).

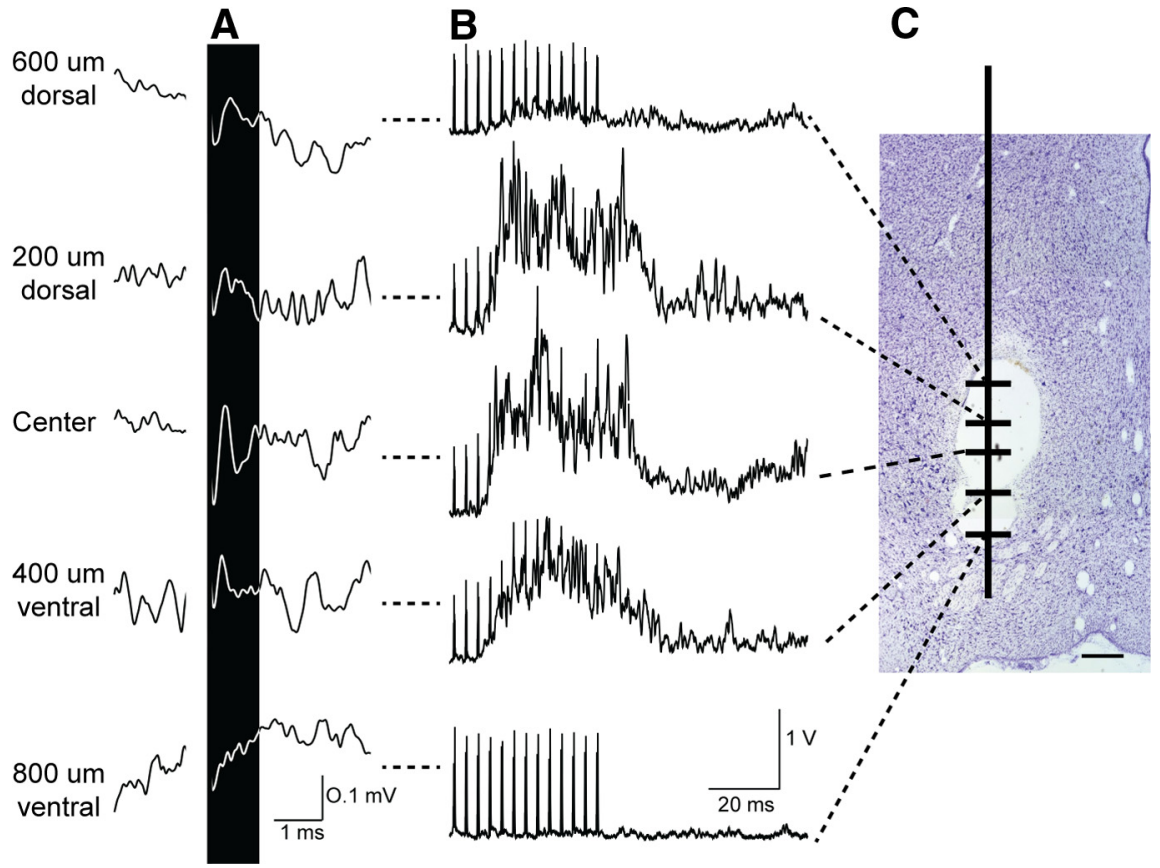

Figure 1. Initial experiments to confirm coordinates for RN microstimulation and injections of anterograde tracers in young animals. $\boldsymbol{A}$, Depth profile of antidromic field potentials recorded and averaged across RN depths in a 6-week-old kitten in response to surface stimulation on the dorsolateral funiculus at C2 (monophasic, $200 \mu \mathrm{A}$ ). The largest field potential (black highlight) EMGs rectified and averaged in response to RN microstimulation at corresponding depths shown in $\boldsymbol{A}$. C, Midbrain coronal sections confirming marking lesions within the RN (Nissl stain). Scale bar, $0.5 \mathrm{~mm}$.

BDA-ChAT; AF488DA-ChAT; BDA-SYN; AF488DA-SYN; BDA-SYN-ChAT; and AF488DA-SYN-ChAT. The final concentrations used produced optimal labeling at all ages examined. No differences significant were noted between the three fluorophores.

Topography of RST terminations. We used a quantitative method for determining the topographic distribution of label within the gray matter in the cervical segments C8-T1 (Brus-Ramer et al., 2007; Friel and Martin, 2007). BDA- and AF488DA-labeled RST axons were traced from transverse spinal sections at $400 \times$ magnification using Neurolucida $(\mathrm{Mi}-$ croBrightField). Varicosities are defined as punctate axonal swellings with a diameter of more than three times the diameter of the nonvaricose axon. Varicosities along axons were marked, and care was taken to verify that axon varicosities were not kinks or other irregularities in the axon. RST axon varicosities are putative presynaptic sites (i.e., boutons) because, as with the CST, they colocalize synaptophysin (Meng et al., 2004) and appose PSD95-containing postsynaptic sites (Friel et al., 2012). For each cat, the axon label (BDA or AF488DA) in the lateral component of the intermediate zone (V, VI, VII) and all of lamina IX in the ventral horn was traced for two sections. Tracings of axon label and varicosities were separately exported and quantified further using Neurolucida or using a suite of programs written in Matlab (MathWorks). For the Matlab analyses, we constructed color-coded density maps of the label. Briefly, a program divided the gray matter into $10 \times 10 \mu \mathrm{m}$ square regions of interest. For each region, we computed the mean density of traced axons 
or marked varicosities. All axonal tracings and varicosities were 1 pixel wide. This corresponds to the amount of label (i.e., total number of pixels) in the region divided by the area of the region. A matrix of mean axon or varicosity density was generated in Matlab that preserved the mediolateral and dorsoventral dimensions of the distribution of label in the gray matter. At the same time, the borders of the gray matter were traced (100× magnification) to obtain a fiducial correspondence between labeling and spinal cord structure. We quantified the regional axon and varicosity density to generate maps of regional distribution of axonal label and to quantify the amounts of label in lamina X (Rexed, 1954). Density is represented according to a color scale, from the lowest density (blue) to the highest (red). Regional distribution maps were generated for individual animals, and averaged maps for all animals within a particular age or treatment group. For the averages, we aligned the data from different animals at the lateral margin of lamina V (Rexed, 1954), where the dorsolateral funiculus forms a wedge-shaped insertion between the gray matter of the dorsal and ventral horns.

Images were acquired with brightfield and epifluorescence on an Olympus BX-60 microscope using $10 \times$ (numerical aperture, 0.3 ), $20 \times$ (numerical aperture, 0.5 ), and $40 \times$ (numerical aperture, 0.75 ) magnification objectives and a Microfire-CCD camera (Optronics) mounted on the microscope. For epifluorescence, a 460-500 $\lambda$ excitation filter and a $510-560 \lambda$ emission filter were used for FITC, and a 535-550 $\lambda$ excitation filter and a 610-675 $\lambda$ emission filter were used for Cy3. To adjust contrast and brightness, Adobe Photoshop (Adobe Systems) was used. A Zeiss LSM 510 confocal microscope was used to visualize triple-labeling (649 $\lambda$ Cy5 excitation filter, $666 \lambda$ emission filter) and for generating optical slices for projection images to verify colocalization of tracers and other markers of interest. When comparing images, all capture and adjustment parameters were kept identical.

Statistics. We used the program SPSS (21.0) or Prism (5.0) for the Macintosh computer to determine the statistical significance of agerelated differences. Motor mapping and anatomical data were compared using ANOVA with Tukey's post hoc test. A two-sample KolmogorovSmirnov test was used to compare the cumulative distribution datasets. Correlation analyses were performed with Pearson's $r$ (two-tailed) or linear regression with ANCOVA to detect differences in slopes. The graphs plot the mean \pm SEM unless specified otherwise.

\section{Results}

The objective of this study was to characterize the motor organization of the rubrospinal system from early postnatal development to maturity. Experiments were conducted on 35 cats ( 9 d of age to adult; Table 1). The RN motor map was examined in 20 animals, and the RST in 19 animals. Some of the animals received tracer injection 2-4 weeks before a terminal RN mapping experiment.

\section{RN identification in immature animals}

Volumetric studies indicate that the size of the red nucleus more than doubles from PW2 to PW8.5 and does not attain the adult volume until PW17 (Villablanca et al., 2000). Furthermore, the location of the RN changes during development (Villablanca et al., 2000). To ensure that we were examining the red nucleus and not the nearby reticular formation in the stimulation and tracing experiments, we modified the suggested stereotaxic coordinates (Rose and Goodfellow, 1973) according to results of three complimentary methods in pilot experiments. First, we identified the locations of antidromic fields of RST neurons in the RN in response to surface stimulation of the spinal cord at C2 (Fig. 1A). Second, in the same session, and at corresponding depths, we electrically stimulated the RN to evoke responses from the extensor carpi radialis muscle (Fig. $1 B$ ). The largest antidromic fields and amplitudes of evoked EMG activity corresponded to the same depths (Fig. 1A,B). Third, at the conclusion of both the initial stimulation experiments to identify the RN in young animals and the mapping studies, marking lesions were made and

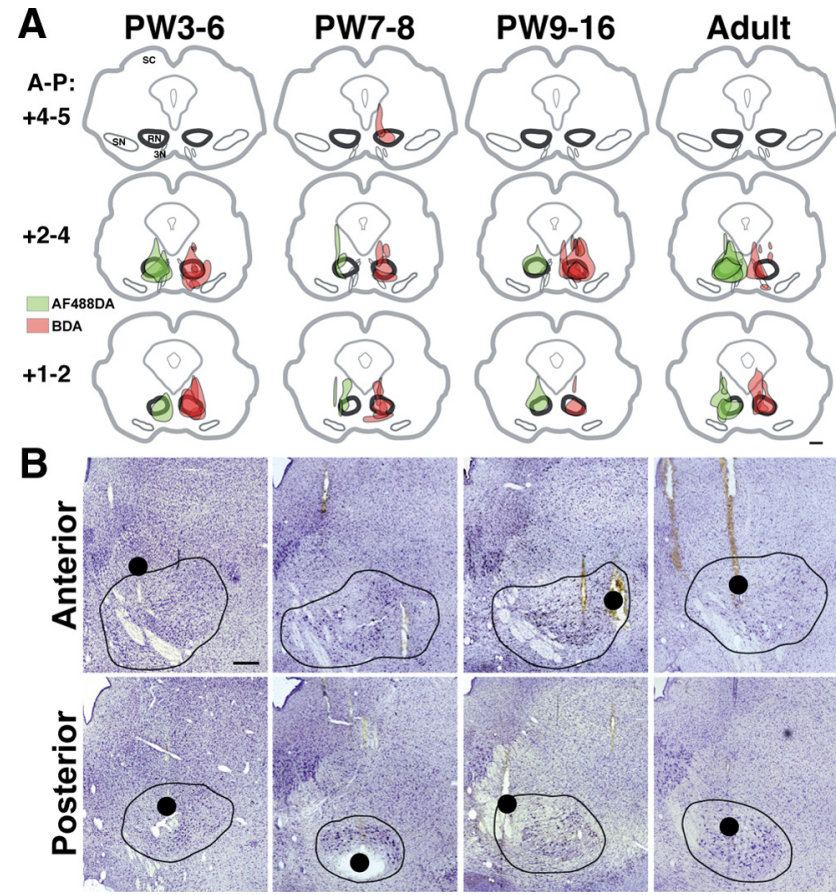

Figure 2. $\quad \boldsymbol{A}$, Reconstruction of tracer injection sites in the midbrain. The regions of maximal amount of injected tracer were located in the posterior two-thirds of the RN and did not vary systematically between animals and age groups. Shown are individual cases for each age group superimposed at caudal, intermediate, and rostral planes in coronal sections $(+1-2,+2-4$, and $+4-6 \mathrm{~mm}$, respectively; Berman, 1968). $\boldsymbol{B}$, Histological reconstruction of stimulating electrode tracks. Nissl sections confirm electrode tracks and marking lesions (red dots, as in Fig. 3) within the anterior (mainly parvocellular; top) and posterior (mainly magnocellular; bottom) RN regions. Note that forelimb movements could be evoked throughout the RN poles. We verified that that ineffective tracks in PW3 animals adequately sampled the RN (bottom left). Midbrain contours show the cytoarchitectural boundaries of the RN outlined in black. Ages are aligned as in $\boldsymbol{A}$. Scale bars: $\boldsymbol{A}, 1 \mathrm{~mm} ; \boldsymbol{B}, 500 \mu \mathrm{m}$. SC, Superior colliculus; SN, substantia nigra; 3N, third nerve.

confirmed histologically that the electrode placements were within the core (magnocellular) of the RN (Fig. 1C). Figure 2 summarizes the locations of all tracer injections $(A)$ and presents representative histology showing marking lesions made after the microstimulation experiments $(B)$. As shown, all tracer injections targeted the red nucleus with minimal variability. Furthermore, a marking lesion provides a histological reference for the stimulation-evoked motor map. In the following sections, we first present results from the RN mapping experiments and then the anatomical findings.

\section{Protracted development of $\mathrm{RN}$ forelimb representation}

To investigate the development and organization of the RN movement representation we used microstimulation (see Materials and Methods). We tested sites with high-resolution sampling (200 $\mu \mathrm{m}$ intervals) throughout the RN. Stimulation at nearly all of the effective sites evoked movement of single contralateral limb joints or trunk movement. Often stimulation evoked ipsilateral eye movement by direct activation of the oculomotor nerve (Ghez, 1975). Whereas we looked for forelimb multijoint effects occurring within $10 \mu \mathrm{A}$ of the threshold for each site, there were very few such responses at any age (data not shown). Nonforelimb movements such as hindlimb, trunk, neck, and face (ipsilateral eye, vibrissae, jaw) movements were also observed. The nonforelimb responses provided information regarding the "effective zone" of the RN to ensure thorough sam- 


\section{PW5-6}

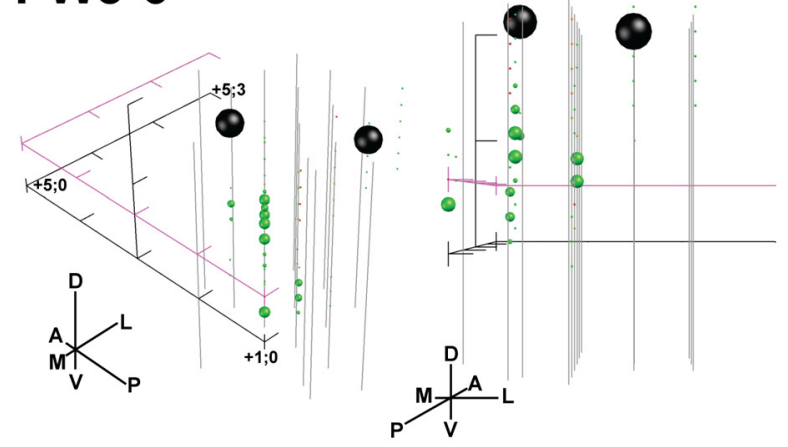

PW7-8

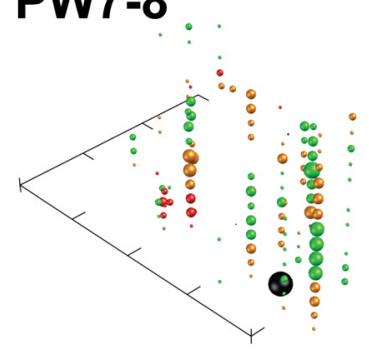

PW9-16
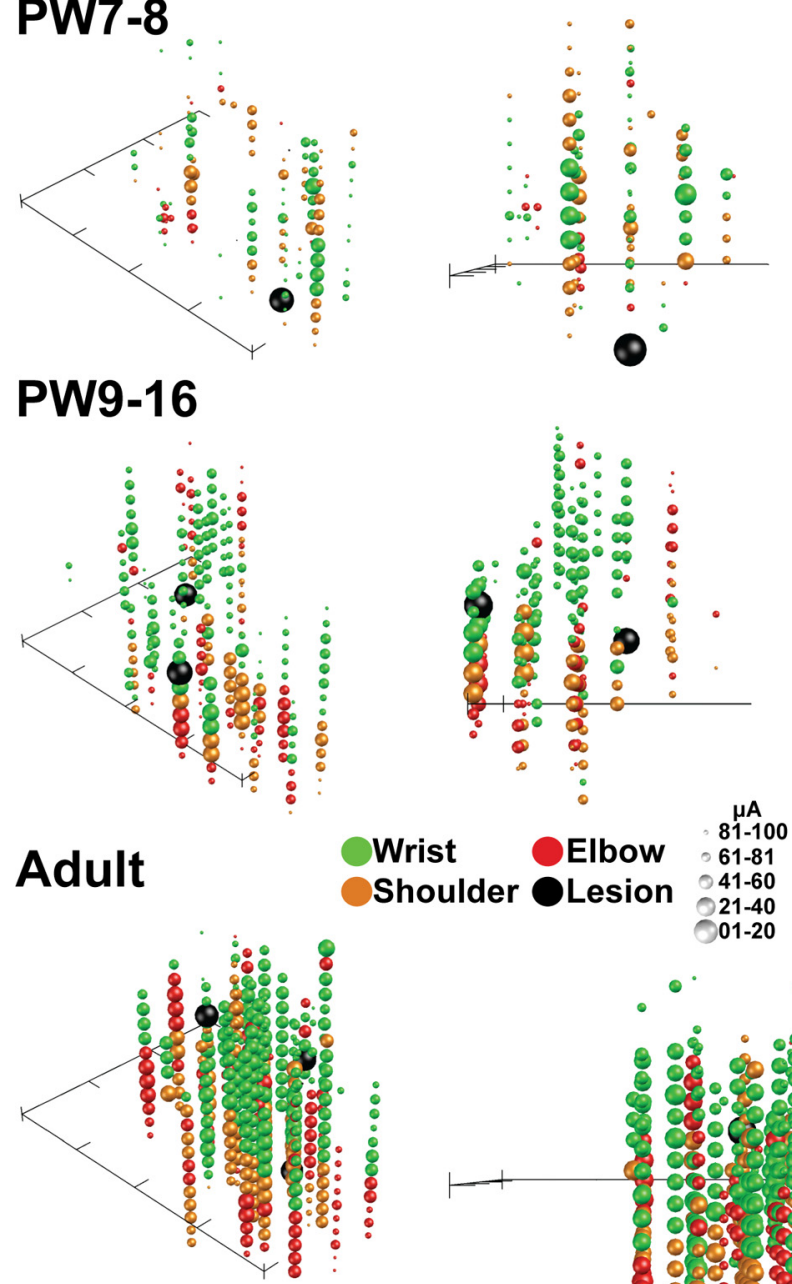

$21-40$

$01-20$

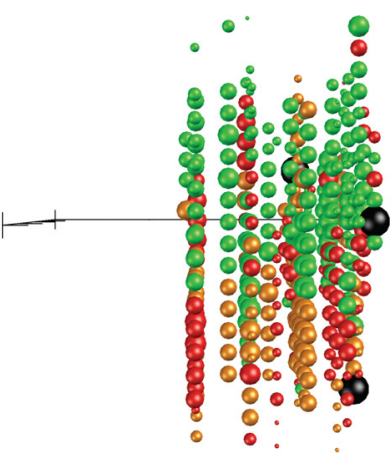

Figure 3. Age-dependent expansion of the RN motor representation. Shown are 3D maps of the RN motor representations from two viewpoints: bird's eye (left) and coronal (posterior to anterior; right). Three representative cases from each age group were combined for comparisons. There was a general topography of distal movements from dorsal sites and proximal movements from intermediate and ventral sites. Hindlimb sites were typically located ventrally (data not shown). Each sphere represents an effective microstimulation site coded by color for joints (green, wrist; red, elbow; orange, shoulder). The size of each sphere was plotted inversely to the threshold value (largest spheres have the lowest thresholds; $20 \mu \mathrm{A} / \mathrm{bin}$ ). Forelimb effects evoked above $100 \mu$ A were plotted for the PW5-PW6 group only. For combining data, the origin of the axis describing the locations of electrode tracts and effective sites for each animal was the same ( $x$-axis, AP, $1-4 \mathrm{~mm}$; $y$-axis, ML, $0-3 \mathrm{~mm}$ ), with the $z$-axis (dorsoventral) set to zero based on the center of the adult RN ( $-2.5 \mathrm{~mm}$ from interaural plane). Axis ticks are spaced pling, but were not included in the analysis. Effects from microstimulation sites determined histologically to be outside the cytoarchitectural margins of the RN were excluded. Rarely did such sites evoke forelimb movements, at any age. The effects of microstimulation of the RN are most likely due to RST activation and not activation of M1 collaterals. Ghez (1975) reported that ablation of M1 did not affect the RN map in adult cats. Furthermore, as we show below, the effects of RN stimulation during early development in the cat are distinctly different from those of M1 stimulation at the same ages.

The youngest age investigated was PW2 $(N=1)$, and stimulation could evoke ipsilateral eye movements, but not forelimb (or hindlimb) movements, even with high currents (up to 200 $\mu \mathrm{A})$. Ipsilateral eye movements could be evoked with single pulses at all ages investigated. Microstimulation at PW3 ( $N=3$ of 3 cats) was also ineffective in evoking forelimb (or hindlimb) movements. Whereas we first observed evoked forelimb movements at PW4, albeit at high currents, activation of all forelimb joints was consistently observed at PW5. For quantitative group analyses, we included only ages when consistent forelimb responses were observed (i.e., PW5 or later).

Three-dimensional plots of the forelimb representation are shown in Figure 3 (left, "bird's-eye" view; right, coronal view). Only sites anatomically reconstructed to be located in the RN are plotted in this figure, and further analysis was completed only for sites anatomically identified to be located in the RN. Importantly, all forelimb joints are represented at all ages, even at PW5, although sparse. Subsets of electrode marking lesions are also plotted (large red spheres) demonstrating localization within the RN (for histology, see Fig. 2B). The plots demonstrate a general distal-to-proximal forelimb joint arrangement as the electrode is moved ventrally, with the hindlimb typically represented ventral to the forelimb, and are consistent with other studies using a similar approach (Ghez and Kubota, 1977; Larsen and Yumiya, 1980). This did not change with age.

\section{RN map expansion during postnatal development}

As indicated above, we verified anatomically that sites were located within the cytoarchitectural boundary of the RN. Anatomical localization within the RN boundaries of sites evoking forelimb movement (Figs. 2B) enables us to analyze rigorously changes in the percentage of sites where stimulation evoked a movement during development (termed "effective sites"). We used the percentage of effective sites as a metric for evaluating motor map development (Chakrabarty and Martin, 2000). We first performed a linear regression analysis of effective site data from all animals between PW3 and PW16 and found a significant correlation with age (Fig. 4A; two-tailed Pearson's $r=0.93, p<$ $0.0001)$. Age-related increases in RN motor excitability explained a significant and substantial portion of the variance $\left(R^{2}=0.86\right)$. For comparison, we replotted motor cortex map development data from our previous study (Chakrabarty and Martin, 2000). Development of the motor cortex map is shifted later in time, by several weeks [age intercept (i.e., $x$-axis) for RN, $12.2 \mathrm{~d}$; for M1, $36.3 \mathrm{~d}$ ], and is more rapid than that of the RN map; the slopes are

at $1 \mathrm{~mm}$ intervals. Additional axes (pink) were added for the PW5-PW6 group to approximate the Z-plane at the RN center (relative to adults) and the dorsoventral extent used for inclusion criteria (data not shown for other ages). Tracks that were ineffective in evoking forelimb movements are shown as solid gray lines for the PW5-PW6 group only, to illustrate adequate sampling within the relatively unresponsive RN in the youngest animals. Marking lesions were made for histological verification of effective sites (large black spheres; Fig. 2B). 
A

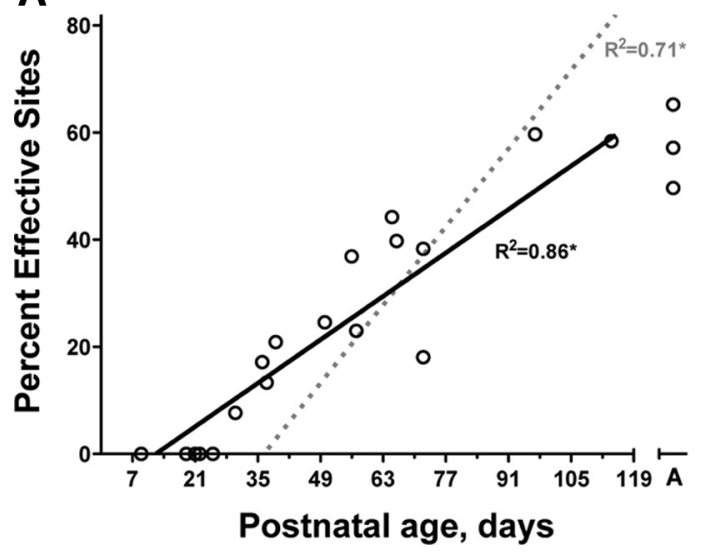

B

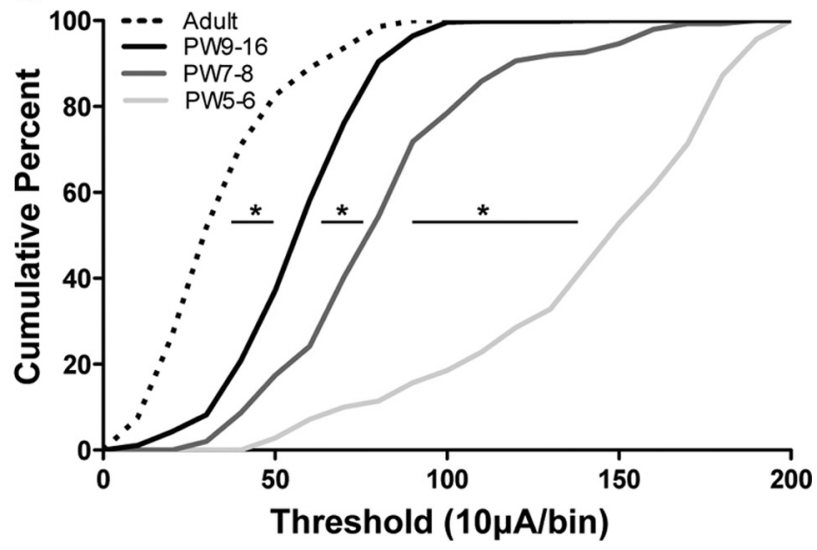

Figure 4. Protracted development of the RN motor map and motor excitability. $\boldsymbol{A}$, Age-dependent increases in the percentage of effective sites for individual cases (black line; open circles). The linear fit is shown as a solid black line $(Y=0.66 x-10.03)$. Also shown for comparison is the linear fit for effective sites in the motor cortex [gray dotted line $(Y=1.54 x-68.62)$; data replotted from Chakrabarty and Martin (2000)]. B, Cumulative frequency plots of movement thresholds show that increased excitability of the rubrospinal system continues to mature even after PW9-PW16. The largest shift toward increased excitability of the rubrospinal system occurred between PW5-PW6 and PW7-PW8. Comparison of the distributions showed significant differences between PW5-PW6 and PW7-PW8 (Kolmogorov-Smirnov test; $D=0.64)$, PW7-PW8 and PW9-PW16 $(D=0.39)$, and PW9-PW16 and adult $(D=0.51)$. Asterisks indicate that the adjoining distributions were significantly different $(p<0.001)$.

A

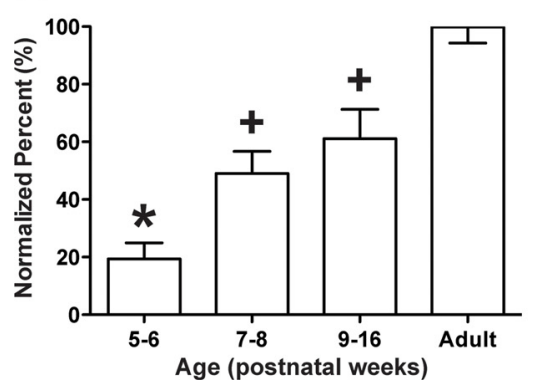

B

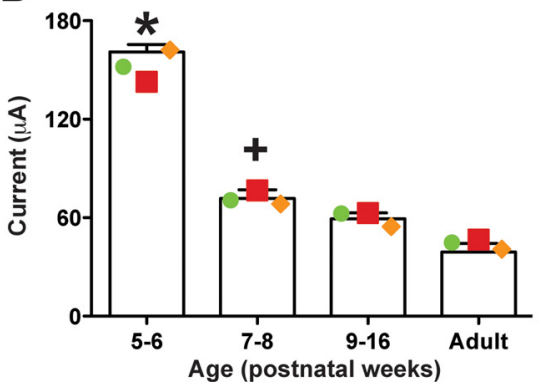

C

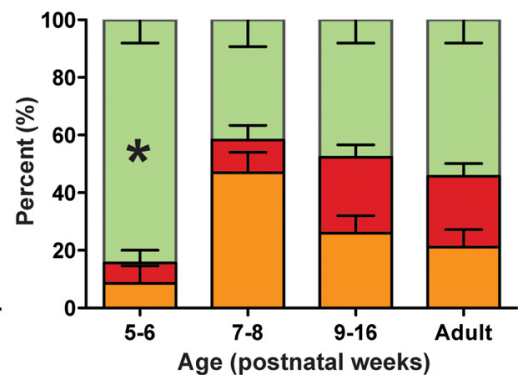

Figure 5. Changes in thresholds and percentages of evoked forelimb movements are age dependent. $\boldsymbol{A}$, The number of effective sites, normalized to adult values, increased during development. Post hoc comparisons revealed that the PW5-PW6 group had fewer effective sites than the PW7-PW8 group and the PW9-PW16 group compared to adults. Additionally, the PW7-PW8 and PW9-PW16 groups had fewer effective sites compared to adults. B, There was a sharp decrease in threshold for wrist, elbow, and shoulder between PW5-PW6 and PW7-PW8. Post hoc comparisons revealed that the PW5-PW6 group had higher thresholds than the PW7-PW8 group, the PW9-PW16 group, and the adult group ( $p$ values $<0.05$ ). The PW7-PW8 group had higher thresholds compared to the adult group ( $p<0.05$ ). Furthermore, post hoc tests indicated that each joint showed significantly higher thresholds until PW9 -PW16 ( $p<0.05$ ). C, The stacked distributions of joint movements for each age group show an overrepresentation of wrist at PW5-PW6, but a more balanced representation of the other forelimb joints thereafter ( $p<0.05$ ). Post hoc comparisons revealed that the PW5-PW6 group had a higher percentage of wrist representation compared to the PW7-PW8 group, the PW9 -PW16 group, and the adult group ( $p$ values $<0.05$ ). Asterisks indicate a difference from all other age groups; plus signs indicate a difference from adults.

significantly different (ANCOVA, $F_{(3,62)}=13.90, p<0.0001$ ). Cumulative frequency distributions of current thresholds for evoking responses from the RN show a progressive shift toward greater numbers of effective sites at lower stimulation currents during development and into adulthood (Fig. 4B). Importantly, the shift toward increased rubrospinal efficiency was greatest between PW5-PW6 and PW7-PW8.

Our subsequent analyses are restricted to those ages in which forelimb responses were evoked by $\mathrm{RN}$ microstimulation (Fig. 5). There was a developmental increase in the percentage of microstimulation sites that were effective to evoke forelimb movements (Fig. $5 A$; normalized to adult; $F_{(3,12)}=24.85, p<0.0001$; post hoc statistics are listed in the figure legend). There were significant differences in mean current intensities between age groups (Fig. $\left.5 B ; F_{(3,11)}=98.42, p<0.0001\right)$. Figure $5 B$ also compares the thresholds for single joints. Each joint showed age-related differences (wrist, $F_{(3,11)}=34.07, p<0.0001$; elbow, $F_{(3,9)}=87.43, p<$ 0.0001 ; shoulder, $\left.F_{(3,10)}=31.80, p<0.0001\right)$. Further analysis of the motor representations revealed a developmental change in the percent of microstimulation sites evoking elbow, shoulder, and wrist joint movement (Fig. $5 C ; F_{(3,11)}=6.60, p<0.01$ ). Importantly, there was an overrepresentation of the wrist at PW5-PW6. Our findings show a parallel age-dependent increase in the percentage of effective sites in the RN producing a motor response and a decrease in the threshold to evoke responses at each joint. Importantly, all joints are represented at all ages.

\section{RST terminations to C8-T1 ventral horn, but not the} intermediate zone, are refined during early postnatal development

To determine how the changes in the organization of RST terminations could underlie RN motor map development, we next traced connections from the RN to the contralateral spinal cord (for injection site locations, see Fig. 2A). The cat RST projects predominantly to the intermediate zone and, additionally, to the motor pools of the cervical enlargement (Holstege, 1987; McCurdy et al., 1987). We determined whether there were age-related differences for RST axons in the intermediate zone and ventral horn. Whereas there may have been subtle quantitative differences, surprisingly the general topography of RST axons in the intermediate zone did not change 


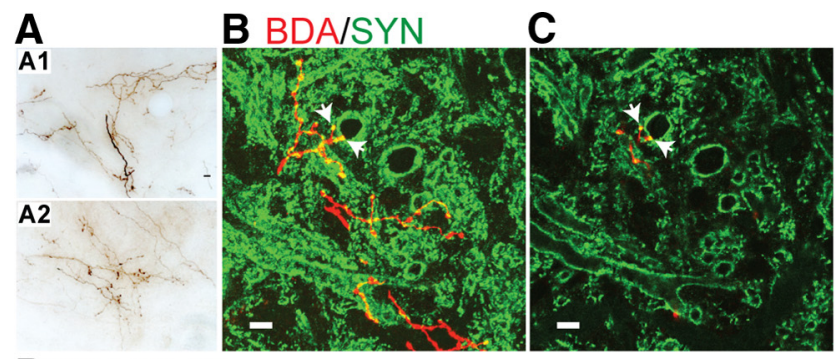

\section{DBDA/ChAT}
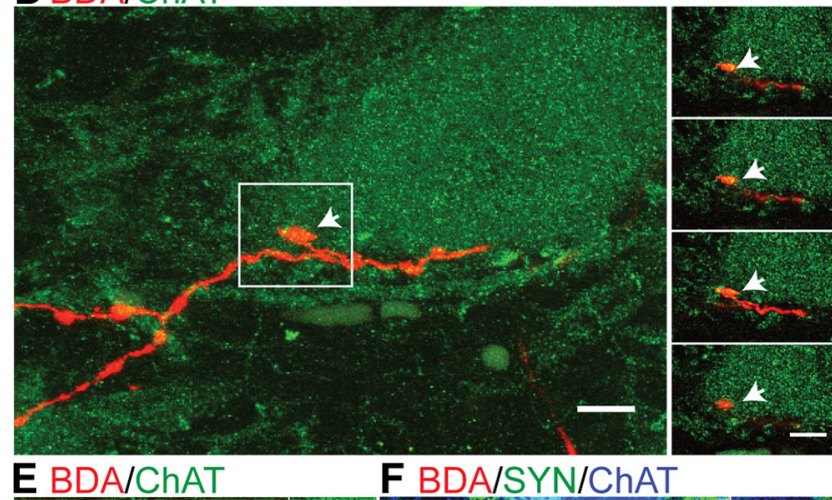

F BDA/SYN/ChAT

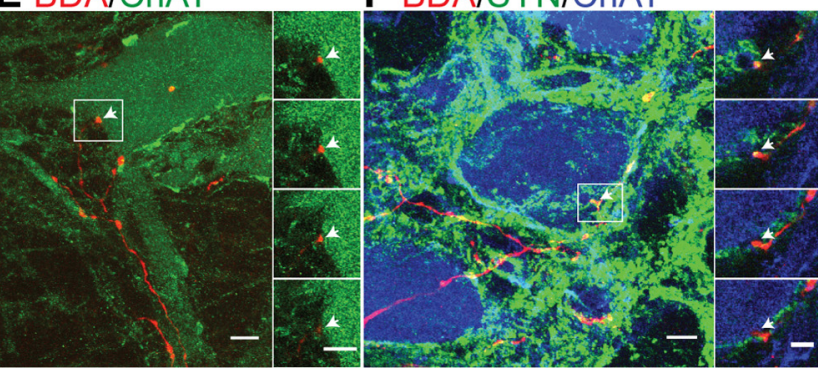

Figure 6. RST projections to the intermediate zone and ventral horn. $\boldsymbol{A}$, Brightfield micrographs of the intermediate zone (lamina VII) show similarly dense label of RST axons and terminations in immature and mature animals ( $\boldsymbol{A} \mathbf{1}, \mathrm{PW} 5 ; \boldsymbol{A} \mathbf{2}$, adult). $\boldsymbol{B}, \boldsymbol{C}$, Double-label confocal images show axon varicosities (selected examples marked with arrows) on RST axons (BDA, red) that colocalize the presynaptic marker synaptophysin (SYN, green) in a PW8 cat (B, Z-stack projection image of 12 optical slices; $\boldsymbol{C}$, single $1 \mu \mathrm{m}$ optical slice). $\boldsymbol{D}, \boldsymbol{E}$, Representative confocal images of lamina IX in a PW8 cat showing numerous RST axons (BDA, red) and varicosities (arrows) in apposition to the soma of motoneurons (ChAT, green). $\boldsymbol{F}$, Apposition of RST axon terminals on motoneurons (ChAT positive) colocalize synaptophysin (PW5; triple label, BDA, red; SYN, green; ChAT, blue). Projection images are shown to the left $(\boldsymbol{D}, \boldsymbol{E}, 12$ optical slices; $\boldsymbol{F}, 11$ slices; each optical slice was $1 \mu \mathrm{m}$ thick) with insets showing serial $1 \mu \mathrm{m}$ optical slices from the boxed regions. The youngest age that RST varicosities (or axons) were found apposing motoneurons $(\boldsymbol{F}$, inset, arrow) and colocalized with the presynaptic marker synaptophysin was PW5. Scale bars: $\boldsymbol{A}-\boldsymbol{E}, 10 \mu \mathrm{m} ; \boldsymbol{F}$, inset, $5 \mu \mathrm{m}$.

between PW3 and maturity. There was an abundance of RST axons throughout the intermediate zone from 3 weeks onward (Fig. 6A). Furthermore, RST axon varicosities colocalized the presynaptic marker synaptophysin at early ages (Fig. $6 B, C$ ), suggesting that these are functional presynaptic sites.

In contrast to the intermediate zone, where labeling is present at PW3, before week 5 we did not observe any RST projections in lamina IX after an extensive search at C7, C8, and T1. Beginning at PW5 we observed motor pool projections and rubromotoneuronal contacts. We examined the development of direct rubromotoneuronal projections using confocal microscopy. Representative confocal projection images (Fig. 6D, E; Z-stack of 12 serial $1 \mu \mathrm{m}$ optical slices) from an PW8 cat show contacts between the RST (red) and a ChAT-labeled motoneuron (green) cell body. Individual adjacent optical slices are shown in the insets of Figure 6, $D$ and $E$ (right). We routinely observed many punc-

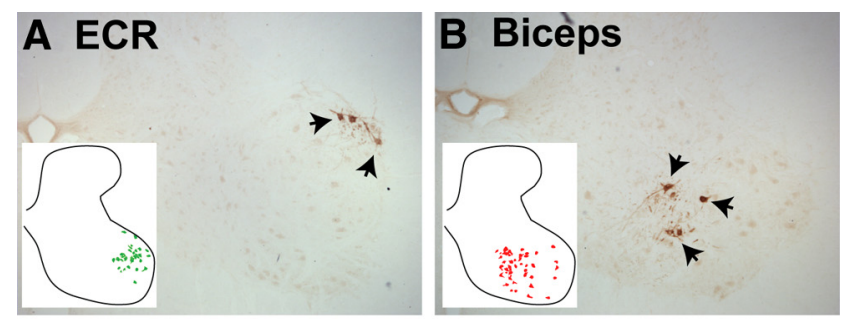

\section{C1 Axon C2 Varicosity C3 Density PW3-6}

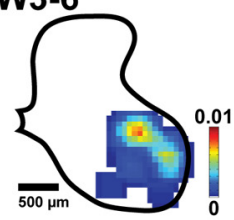

PW7-8

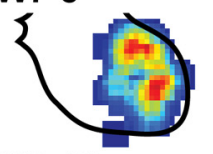

PW9-16
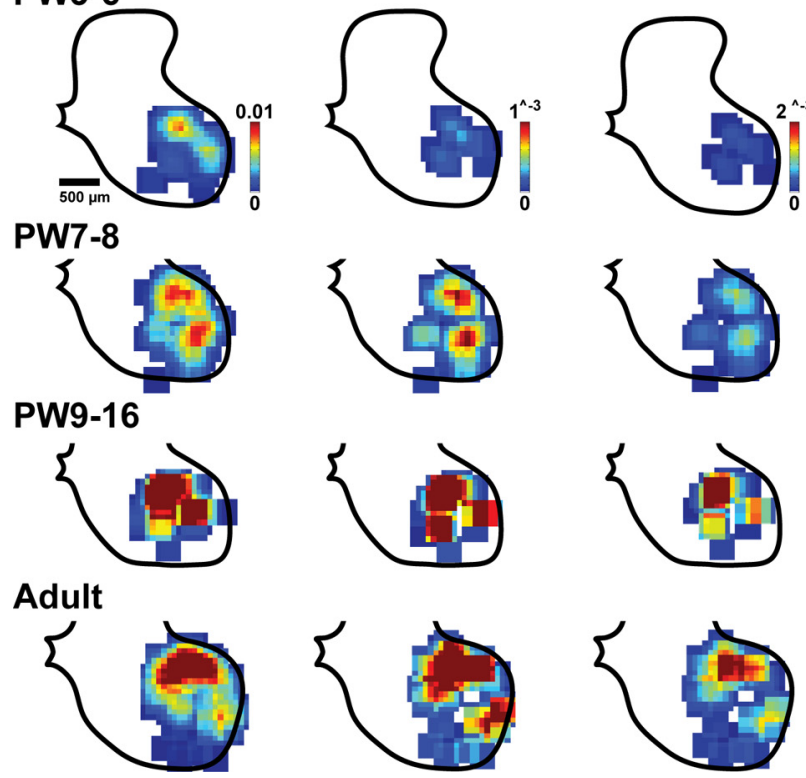

Figure 7. Late development of RST projections to cervical motor pools. A, B, Brightfield micrographs of retrogradely labeled motoneurons (arrows) for the extensor carpi radialis $(\boldsymbol{A})$ and bicep $(\boldsymbol{B})$ muscles in a representative adult. The insets plot motoneurons cell bodies traced and merged across multiple sections from caudal C7 to rostral T1 segments in two adult animals. C1-C3, Heat maps of RST topography in the motor pools ( $\mathbf{C}$, axons; $\mathbf{C}$, varicosities; $\mathbf{C}$ varicosity density; i.e., density matrices of (2 divided by density matrices of (1). At PW3-PW6 there was sparse axonal and varicosity labeling, and correspondingly low varicosity density that dramatically increased by PW7-PW8. Color scales are the same for all age groups in each column (arbitrary units).

tate RST axon varicosities (arrows) on the soma and proximal dendrites of motoneurons. Furthermore, we found RST axon varicosities colabeled with the presynaptic marker synaptophysin in close apposition to ChAT-labeled motoneuron cell bodies, suggesting functional direct contacts (PW5 animal; Fig. 6F).

We next asked whether there was a postnatal development of the distribution of RST projections to the motor pools. We retrogradely labeled two key forelimb muscles, the bicep brachii and ECR, which mediate the most common kinematic changes produced by $\mathrm{RN}$ microstimulation (Fig. 3). The ECR motor pool is located dorsolaterally, as shown for a representative adult cat (Fig. 7A). The inset plots the location of motoneuron cell bodies across sections from C7 to T1. Labeled bicep motoneurons are commonly located ventromedial to the ECR motor pool or more broadly distributed within lamina IX (Fig. $7 B$, inset). The heat maps (Fig. 7C) show the distribution of RST axons and putative synapses $(\mathrm{C} 1, \mathrm{C} 2)$ in the ventral horn for four age groups $(n=4$ animals/age group). Each pixel is the average value within a $10 \times 10$ $\mu \mathrm{m}$ region of interest. The $X-Y$ matrix for the varicosity distribution was divided by the matrix for the axon distribution to reveal a regional axon varicosity density map (C3). These data show agerelated increases in the density and/or extent of motor pool RST axons, putative synapses, and the density of putative synapses. 

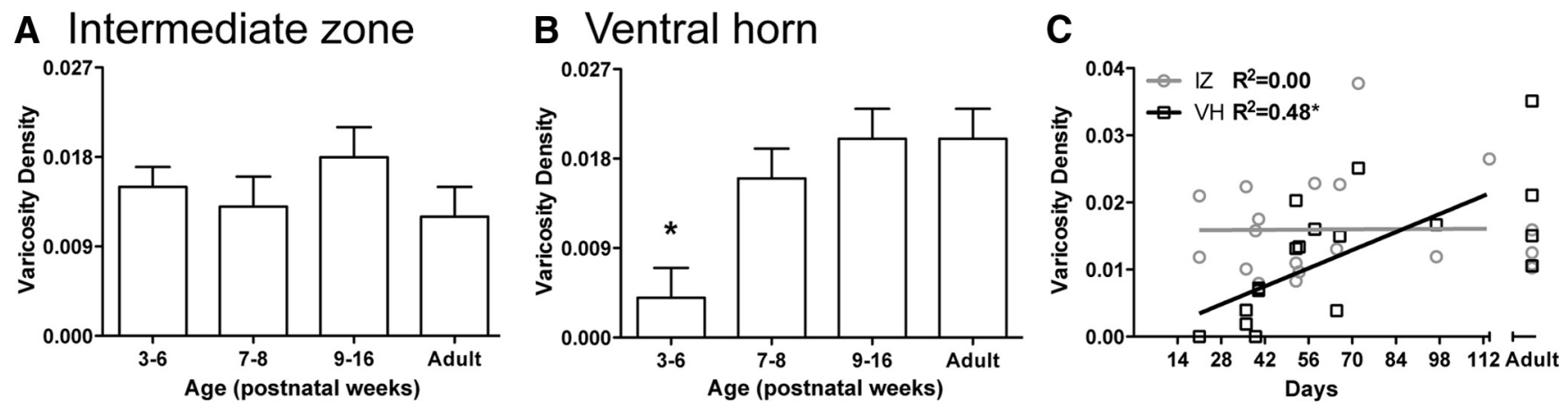

Figure 8. Quantification of rubrospinal terminations. $\boldsymbol{A}$, There was no age-dependent change in density of presumptive presynaptic terminals (varicosities) in the intermediate zone (laminae V-VII). B. Conversely, there was a significant increase in varicosity density of RST terminations in the motor pool region (lamina IX). Post hoc comparisons revealed that the PW3-PW6 group had lower axon varicosity densities compared to the PW7-PW8 group, the PW9-PW16 group, and the adult group ( $p$ values $<0.05$ ). Asterisks indicate different from all other groups. $C$, A linear fit was found to be significant for the ventral horn $\left(\mathrm{VH} ; Y=0.000192 x-0.00058 ; R^{2}=0.48 ; p=0.002\right)$, but not the intermediate $z 0 n e\left(I Z ; Y=0.0 x+0.0158 ; R^{2}=0.00, p=0.96\right)$.

We quantified changes in the local density of RST varicosities in the intermediate zone and ventral horn. As the morphology suggested, there were no age-dependent changes in the density of RST intermediate zone varicosities (Fig. $8 A ; F_{(3,15)}=0.90, p=$ 0.46). Furthermore, the correlation was low $(r=0.33)$ and not significant (Fig. $8 C$, gray data points and line). By contrast, we found a significant age-dependent increase in varicosity density within lamina IX (Fig. $8 B ; F_{(3,15)}=11.42, p<0.001$ ). Regression analysis for kittens between PW3 and PW16 (i.e., ungrouped) revealed a significant correlation $(r=0.73, p=0.001)$, and the linear relationship explained about half $(48 \%)$ of the variance (Fig. 8C, black data points and line). Our findings show that, between PW3 and PW16 there is an age-related increase in rubrospinal tract varicosities (i.e., putative synapses) in the motor nuclei, but not in the intermediate zone.

\section{Discussion}

Motor skills in kittens begin to mature at $\sim$ PW6, rapidly develop until PW8, and increase in complexity at a slower pace into adolescence (PW12). It has been shown that the frequency of paw contacts with objects (toys) in the environment starts to rapidly increase at PW6 and begins to plateau at $\sim$ PW8 (Barrett and Bateson, 1978). This is the age when kittens begin making visually guided targeted movements with the forelimbs. Upon this background of motor development, we examined the maturation of the RN motor map and RST projections to the intermediate zone and ventral horn. The RN forelimb motor map is present at PW5, when there is a distal representation, and undergoes rapid development by PW7/PW8. This early maturation pattern contrasts sharply with that of M1 in the kitten, where the forelimb motor map does not begin to develop until PW7-PW8 (Chakrabarty and Martin, 2000). And from this age onward, the map develops from proximal to distal. Our findings support an early motor function of the RN during the developmental phase when distal limb skills are being refined.

We found surprising anatomical correlates of RN map development. RST projection density was mature in the intermediate zone at PW3, which is before the map is present, but was scarce in the motor pools. As the RN motor map developed, so too did the RST motor pool terminations. Thus, development of RST terminations in the motor pool, not intermediate zone, paralleled RN motor map development. Remarkably, RST development is opposite to that of the CST, where there is an age-dependent development of intermediate zone terminations and elimination of transient motor pool projections (Li and Martin, 2000, 2001,
2002). Our findings suggest two phases of functional maturation of the lateral motor system, a "precorticospinal" phase dominated by the rubrospinal system that plays a role in distal and proximal limb coarse control, and a second "cocorticospinal" phase relying on both lateral pathways that is important in the development of limb skills.

\section{Protracted development of the RN motor map}

The RN motor map from PW5 onward, which was the age when there were sufficient numbers of effective sites to infer somatotopy, comprised a dorsal-to-ventral and distal-to-proximal organization, similar to the mature RN (Ghez, 1975; Larsen and Yumiya, 1980). Both the RN and M1 motor maps appear to spatially represent adjoining limb segments (Donoghue et al., 1992). Further analysis showed that although there was a general limb somatotopy in the RN, there was also some mixing of joint representations, usually of the adjoining joint. Thus, like M1, the RN appears to have a mosaic distribution that could help coordinate joint synergies. There was a clear overrepresentation of the wrist at PW5, which could mediate early distal control, during the precorticospinal phase. After PW7, each joint's contribution to the forelimb map was more uniform. This change may reflect cocorticospinal use-dependent rebalancing of the forelimb motor representations (Martin et al., 2005), for example, from repetitive wrist use for kneading milk expression during nursing to the coordinated use of all forelimb joints as play behavior with littermates dominates (PW7, after weaning; Martin and Bateson, 1985).

\section{Development of RST intermediate zone projections}

The RST is a major input to cervical motor circuits from the brain stem, and is primarily directed to distal control. At all postnatal ages RST axon collaterals project from the dorsolateral funiculus into the intermediate zone. Surprisingly, RST projections to the intermediate zone were morphologically mature at PW3. This contrasts sharply with the CST, which shows a protracted development into late adolescence ( $\mathrm{Li}$ and Martin, 2001, 2002). For M1, we demonstrated an important role for activity and limb use in directing the refinement of CST projections to the dorsal horn and intermediate zone (Martin et al., 2004). This fits with the parallel development of CST connections, the motor map, and motor skill. Since the RST intermediate zone projections and RN motor map are present before the onset of skill development, this motor system may depend less on use-dependent refinement and more on guidance factors for the establishment of spinal connections, at least during this precorticospinal period. 


\section{Development of RST motor pool connectional specificity}

There are remarkable differences in development of RST and CST ventral projections in cats that may inform function. The CST has motor pool projections early in development that are subsequently eliminated (Li and Martin, 2000), whereas we have found that the RST has late-developing motor pool projections that further develop into maturity. Thus, as RST neurons are establishing their motor pool projections, CST neurons are eliminating theirs. CST developmental studies in rats show a similar delayed withdrawal from the motor pools (Curfs et al., 1994), and in the monkey, delayed establishment (Kuypers, 1962; Armand et al., 1997). If development of motor pool projections are use- or activity-dependent, then what aspects of developing movements could lead to reciprocal CST and RST changes? Corticomotoneuronal connections in the monkey are regarded to play a role in independent finger movements, inferred from studies showing correlated developmental timing as well as from studies demonstrating CST/M1 lesion impairs individuated control (Lawrence and Kuypers, 1968b; Porter and Lemon, 1993). Corticomotoneuronal connections likely participate in other limb functions, as they are also present on proximal muscle motoneurons, albeit with a lower probability (Fetz and Cheney, 1980; Buys et al., 1986; Lemon et al., 1986, 1987; McKiernan et al., 2000). Since cats do not express independent finger movements and individuated control, we expect that motor development would not support maintenance and further growth of these connections in a usedependent manner. Interestingly, electrical stimulation of the developing CST in kittens maintains its motor pool projections, at least until later in development (Salimi and Martin, 2004), further supporting a role for activity in CST motor pool projections.

Rubromotoneuronal/motor pool projections are highly conserved throughout evolution, present in rodents, cats, and primates (Holstege, 1987; McCurdy et al., 1987; Mewes and Cheney, 1991; Küchler et al., 2002), suggesting common and important functions. The function of rubromotoneuronal connections in monkey is thought to differ from that of corticomotoneuronal connections. Physiological studies demonstrate a greater proportion of rubromotoneuronal cells that cofacilitate extensor and flexor muscles than corticomotoneuronal cells, suggesting that the rubromotoneuronal projection maintains excitability of multiple motoneuron groups and is involved in more synergistic than individuated control (Mewes and Cheney, 1991). This proposed function of rubromotoneuronal connections in the monkey could be shared by cats and rats. Our axon reconstructions suggest that rubromotoneuronal connections collectively innervate proximal and distal forelimb motor pools, consistent with a role in synergistic control. It is intriguing that, in the absence of corticomotoneuronal projections in the cat and late development in the monkey (Kuypers, 1962; Armand et al., 1997), maturation of rubromotoneuronal connections may support the behavioral transition toward dexterous skill.

\section{Relationship between postnatal maturation of the $\mathrm{RN}$ motor map and RST terminations}

There were no evoked movements in the PW3 group, which is surprising considering that the RST exhibits a mature density of varicosities in the intermediate zone at this age. Furthermore, varicosities at young ages colocalize synaptophysin, suggesting that many are competent to release transmitter. RST axon conduction velocity matures during the first few postnatal weeks, and corticorubral connections also mature early, further supporting functional competency (Song and Murakami, 1990; Song et al., 1995). The absence or paucity of effects at and before PW5 ap- pears not because of the generalized spinal circuit immaturity, since reflexes are expressed at even younger ages (Villablanca and Olmstead, 1979). It is plausible that the failure of the immature $\mathrm{RN}$ to evoke movement is due to immaturity of the postsynaptic targets of the RST in the intermediate zone. Although not yet studied for the RST, this mechanism was suggested for the CST, where we found that the spinal cholinergic interneurons that are the targets of the CST develop late (Chakrabarty et al., 2009). We propose that delayed maturation of motoneuronal connections during the precorticospinal period is an important developmental change for establishing the RN motor map. As animals mature, there may also be important functional maturation of RST connections with interneurons, although this would occur without morphological changes. Further experiments need to be conducted to determine whether rubromotoneuronal connections enable the $\mathrm{RN}$ motor map or are a consequence of map development.

It is likely that skilled limb movements - such as visually guided prehension, gait modification, and postural adjustments-require refinement of both the CST and RST, since in maturity both components appear to play a role in their production (Gibson et al., 1985; Cheney et al., 1988; Martin and Ghez, 1988, 1991; Lavoie and Drew, 2002; Drew et al., 2004; Zelenin et al., 2010; Stout and Beloozerova, 2013). Whereas the RST is thought to be reduced in nonhuman primates and humans, compared with cats and rats, it may be important in all species as an early postnatal convener of lateral motor systems functions during the precorticospinal phase, especially for intermediate cerebellar control (Robinson et al., 1987), superseded later in development by the CST.

The RST may take over some motor functions after CST injury (Raineteau and Schwab, 2001; Raineteau et al., 2002). Much less considered is the RST's role in recovery when the developing CST becomes damaged, particularly during the precorticospinal phase. The protracted cocorticospinal phase of RN/RST development, which parallels expression of skilled limb control, may comprise a period when behavior and other activity-dependent processes shape its development. This late period of rubrospinal development, when the capability to drive motor function is established, albeit immature, may provide a protracted window of opportunity to intervene with therapeutic treatments for motor impairments following perinatal injuries, such as stroke.

\section{References}

Armand J, Olivier E, Edgley SA, Lemon RN (1997) Postnatal development of corticospinal projections from motor cortex to the cervical enlargement in the macaque monkey. J Neurosci 17:251-266. Medline

Asanuma H, Sakata H (1967) Functional organization of a cortical efferent system examined with focal depth stimulation in cats. J Neurophysiol 30:35-54.

Barrett P, Bateson P (1978) The development of play in cats. Behaviour 66:106-120. CrossRef

Berman AL (1968) The brain stem of the cat. Madison, WI: University of Wisconsin.

Bruce IC, Tatton WG (1980) Sequential output-input maturation of kitten motor cortex. Exp Brain Res 39:411-419. Medline

Brus-Ramer M, Carmel JB, Chakrabarty S, Martin JH (2007) Electrical stimulation of spared corticospinal axons augments connections with ipsilateral spinal motor circuits after injury. J Neurosci 27:13793-13801. CrossRef Medline

Buys EJ, Lemon RN, Mantel GW, Muir RB (1986) Selective facilitation of different hand muscles by single corticospinal neurones in the conscious monkey. J Physiol 381:529-549. Medline

Chakrabarty S, Martin JH (2000) Postnatal development of the motor representation in primary motor cortex. J Neurophysiol 84:2582-2594. Medline

Chakrabarty S, Shulman B, Martin JH (2009) Activity-dependent codevel- 
opment of the corticospinal system and target interneurons in the cervical spinal cord. J Neurosci 29:8816-8827. CrossRef Medline

Cheney PD, Mewes K, Fetz EE (1988) Encoding of motor parameters by corticomotoneuronal (CM) and rubromotoneuronal (RM) cells producing postspike facilitation of forelimb muscles in the behaving monkey. Behav Brain Res 28:181-191. CrossRef Medline

Curfs MHJM, Gribnau AAM, Dederen PJWC (1994) Selective elimination of transient corticospinal projections in the rat cervical spinal cord gray matter. Dev Brain Res 78:182-190. CrossRef

Donoghue JP, Leibovic S, Sanes JN (1992) Organization of the forelimb area in squirrel monkey motor cortex: representation of digit, wrist, and elbow muscles. Exp Brain Res 89:1-19. Medline

Drew T, Prentice S, Schepens B (2004) Cortical and brainstem control of locomotion. Prog Brain Res 143:251-261. CrossRef Medline

Fetz EE, Cheney PD (1980) Postspike facilitation of forelimb muscle activity by primate corticomotoneuronal cells. J Neurophysiol 44:751-772. Medline

Friel KM, Martin JH (2007) Bilateral activity-dependent interactions in the developing corticospinal system. J Neurosci 27:11083-11090. CrossRef Medline

Friel K, Chakrabarty S, Kuo HC, Martin J (2012) Using motor behavior during an early critical period to restore skilled limb movement after damage to the corticospinal system during development. J Neurosci 32: 9265-9276. CrossRef Medline

Ghez C (1975) Input-output relations of the red nucleus in the cat. Brain Res 98:93-108. CrossRef Medline

Ghez C, Kubota K (1977) Activity of red nucleus neurons associated with a skilled forelimb movement in the cat. Brain Res 131:383-388. CrossRef Medline

Gibson AR, Houk JC, Kohlerman NJ (1985) Magnocellular red nucleus activity during different types of limb movement in the macaque monkey. J Physiol 358:527-549. Medline

Holstege G (1987) Anatomical evidence for an ipsilateral rubrospinal pathway and for direct rubrospinal projections to motoneurons in the cat. Neurosci Lett 74:269-274. CrossRef Medline

Küchler M, Fouad K, Weinmann O, Schwab ME, Raineteau O (2002) Red nucleus projections to distinct motor neuron pools in the rat spinal cord. J Comp Neurol 448:349-359. CrossRef Medline

Kuypers HG (1962) Corticospinal connections: postnatal development in the rhesus monkey. Science 138:676-678. Medline

Kuypers HG (1981) Anatomy of the descending pathways. In: Handbook of physiology, neurophysiology, Vol II (Brookhart JM, Mountcastle VB, eds), pp 597-666. Bethesda, MD: American Physiological Society.

Larsen KD, Yumiya H (1980) The red nucleus of the monkey. Topographic localization of somatosensory input and motor output. Exp Brain Res 40:393-404. Medline

Lavoie S, Drew T (2002) Discharge characteristics of neurons in the red nucleus during voluntary gait modifications: a comparison with the motor cortex. J Neurophysiol 88:1791-1814. Medline

Lawrence DG, Kuypers HG (1968a) The functional organization of the motor system in the monkey. II. The effects of lesions of the descending brain-stem pathways. Brain 91:15-36. CrossRef Medline

Lawrence DG, Kuypers HG (1968b) The functional organization of the motor system in the monkey. I. The effects of bilateral pyramidal lesions. Brain 91:1-14. CrossRef Medline

Lemon RN, Mantel GW, Muir RB (1986) Corticospinal facilitation of hand muscles during voluntary movement in the conscious monkey. J Physiol 381:497-527. Medline

Lemon RN, Muir RB, Mantel GW (1987) The effects upon the activity of hand and forearm muscles of intracortical stimulation in the vicinity of corticomotor neurons in the conscious monkey. J Physiol 66:621-637. Medline

Li Q, Martin JH (2000) Postnatal development of differential projections from the caudal and rostral motor cortex subregions. Exp Brain Res 134: 187-198. CrossRef Medline

Li Q, Martin JH (2001) Postnatal development of corticospinal axon terminal morphology in the cat. J Comp Neurol 435:127-141. CrossRef Medline

Li Q, Martin JH (2002) Postnatal development of connectional specificity of corticospinal terminals in the cat. J Comp Neurol 447:57-71. CrossRef Medline

Martin JH, Ghez C (1988) Red nucleus and motor cortex: parallel motor systems for the initiation and control of skilled movement. Behav Brain Res 28:217-223. CrossRef Medline

Martin JH, Ghez C (1991) Task-related coding of stimulus and response in cat red nucleus. Exp Brain Res 85:373-388. Medline

Martin JH, Choy M, Pullman S, Meng Z (2004) Corticospinal development depends on experience. J Neurosci 24:2122-2132. CrossRef Medline

Martin JH, Engber D, Meng Z (2005) Effect of forelimb use on postnatal development of the forelimb motor representation in primary motor cortex of the cat. J Neurophysol 93:2822-2831. CrossRef

Martin J, Friel K, Salimi I, Chakrabarty S (2009) Corticospinal development. In: Encyclopedia of neuroscience (Squire L, ed), pp 203-214. Oxford, UK: Academic.

Martin P, Bateson P (1985) The ontogeny of locomotor play behavior in the domestic cat. Anim Behav 33:502-510. CrossRef

Massion J (1967) The mammalian red nucleus. Physiol Rev 47:383-436. Medline

McCurdy ML, Hansma DI, Houk JC, Gibson AR (1987) Selective projections from the cat red nucleus to digit motor neurons. J Comp Neurol 265:367-379. CrossRef Medline

McKiernan BJ, Marcario JK, Karrer JH, Cheney PD (2000) Correlations between corticomotoneuronal (CM) cell postspike effects and cell-target muscle covariation. J Neurophysiol 83:99-115. Medline

Meng Z, Li Q, Martin JH (2004) The transition from development to motor control function in the corticospinal system. J Neurosci 24:605-614. CrossRef Medline

Mewes K, Cheney PD (1991) Facilitation and suppression of wrist and digit muscles from single rubromotoneuronal cells in the awake monkey. J Neurophysiol 66:1965-1977. Medline

Porter R, Lemon R (1993) Corticospinal function and voluntary movement. Oxford, UK: Oxford Science.

Raineteau O, Schwab ME (2001) Plasticity of motor systems after incomplete spinal cord injury. Nat Rev Neurosci 2:263-273. CrossRef Medline

Raineteau O, Fouad K, Bareyre FM, Schwab ME (2002) Reorganization of descending motor tracts in the rat spinal cord. Eur J Neurosci 16:17611771. CrossRef Medline

Rexed B (1954) The cytoarchitectonic organization of the spinal cord in the cat. J Comp Neurol 100:297-379. CrossRef Medline

Robinson FR, Houk JC, Gibson AR (1987) Limb specific connections of the cat magnocellular red nucleus. J Comp Neurol 257:553-577. CrossRef Medline

Rose GH, Goodfellow EF (1973) A stereotaxic atlas of the kitten brain. Los Angeles: Brain Information Service/Brain Research Institute, University of California, Los Angeles.

Salimi I, Martin JH (2004) Rescuing transient corticospinal terminations and promoting growth with corticospinal stimulation in kittens. J Neurosci 24:4952-4961. CrossRef Medline

Song WJ, Murakami F (1990) Ipsilateral interpositorubral projection in the kitten and its relation to post-hemicerebellectomy plasticity. Dev Brain Res 56:75-85. CrossRef

Song WJ, Okawa K, Kanda M, Murakami F (1995) Perinatal development of action potential propagation in cat rubrospinal axons. J Physiol 488:419426. Medline

Stout EE, Beloozerova IN (2013) Differential responses of fast- and slowconducting pyramidal tract neurons to changes in accuracy demands during locomotion. J Physiol 591:2647-2666. Medline

Villablanca JR, Olmstead CE (1979) Neurological development of kittens. Dev Psychol 12:101-127. CrossRef

Villablanca JR, Schmanke TD, Crutcher HA, Sung AC, Tavabi K (2000) The growth of the feline brain from fetal into adult life. II. A morphometric study of subcortical nuclei. Brain Res Dev Brain Res 122:21-33. CrossRef Medline

Zelenin PV, Beloozerova IN, Sirota MG, Orlovsky GN, Deliagina TG (2010) Activity of red nucleus neurons in the cat during postural corrections. J Neurosci 30:14533-14542. CrossRef Medline 